\title{
Crystal growth and characterization of the dilutable frustrated spin-ladder compound $\mathrm{Bi}\left(\mathrm{Cu}_{1-x} \mathrm{Zn}_{x}\right)_{2} \mathrm{PO}_{6}$
}

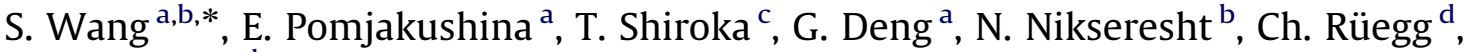 \\ H.M. Rønnow ${ }^{\mathrm{b}}$, K. Conder ${ }^{\mathrm{a}}$ \\ ${ }^{a}$ Laboratory for Developments and Methods, Paul Scherrer Institut, CH-5232 Villigen, Switzerland \\ ${ }^{\mathrm{b}}$ Laboratory for Quantum Magnetism, Ecole Polytechnique Federale de Lausanne, CH-1015 Lausanne, Switzerland \\ ${ }^{\mathrm{c}}$ Laboratorium für Festkörperphysik, ETH Hönggerberg, CH-8093 Zürich, Switzerland \\ ${ }^{\mathrm{d}}$ London Centre for Nanotechnology and Department of Physics and Astronomy, University College London, London WC1E 6BT, United Kingdom
}

\section{A R T I C L E I N F O}

\section{Article history:}

Received 8 April 2010

Received in revised form

24 August 2010

Accepted 28 September 2010

Communicated by R.S. Feigelson

Available online 7 October 2010

Keywords:

A2. Single crystal growth

A2. Travelling solvent zone growth

B1. $\mathrm{BiCu}_{2} \mathrm{PO}_{6}$

B1. Phosphates

B1. Cuprates

B2. Magnetic materials

\begin{abstract}
A B S T R A C T
High quality centimeter size single crystals of $\mathrm{Bi}\left(\mathrm{Cu}_{1-x} \mathrm{Zn}_{x}\right)_{2} \mathrm{PO}_{6}(x=0 \%, 1 \%, 5 \%)$ have been successfully grown by the Travelling Solvent Floating Zone (TSFZ) technique. The crystals were grown with a rate of $1 \mathrm{~mm} / \mathrm{h}$ in a gas mixture of $20 \% \mathrm{O}_{2}$ in Ar. Characterization of the single crystal samples by means of optical microscopy, X-ray powder diffraction, X-ray Laue diffraction, neutron diffraction and magnetization measurement are reported. The magnetic susceptibility $\chi_{\text {spin }}(T)$, measured from 2 to $300 \mathrm{~K}$, passes a broad maximum around $60 \mathrm{~K}$, followed by an exponential decrease towards lower $T$, and confirm the formation of a spin singlet ground state at low temperature. At low-temperature a paramagnetic Curie-like upturn quantitatively reflect the increasing $\mathrm{Zn}$-doping level.
\end{abstract}

(c) 2010 Elsevier B.V. All rights reserved.

\section{Introduction}

Recently, there has been great interest in the magnetic properties of pure and doped low-dimensional and frustrated Heisenberg antiferromagnetic (HAF) spin systems. The interest stems from the variety of intriguing phenomena originating from the quantum fluctuations, and from their possible relevance to high- $T_{\mathrm{c}}$ superconductivity [1-5]. In this context, the even-leg spin ladder is arguably the simplest model exhibiting a singlet ground state with an energy gap to dispersive triplet states. Based on theoretical studies, Dagatto and Rice predicted that, under modest hole doping, spin ladders might become superconducting [6]. Indeed, superconductivity has been discovered in the Ca-doped two-leg ladder system $\mathrm{Sr}_{14-x} \mathrm{Ca}_{x} \mathrm{Cu}_{24} \mathrm{O}_{41}(x=13.6)$ under high pressure of 3.5 GPa [7].

Considerable experimental and theoretical work has been devoted to unravel the effect of non-magnetic dilution of the gapped spin liquid state. For example, in $\mathrm{SrCu}_{2} \mathrm{O}_{3}$ (2-leg) and $\mathrm{Sr}_{3} \mathrm{Cu}_{2} \mathrm{O}_{5}$ (3-leg) systems [8], doping non-magnetic $\mathrm{Zn}^{2+}(S=0)$

\footnotetext{
* Corresponding author at: Laboratory for Developments and Methods, Paul Scherrer Institut, WLGA-U117, CH-5232 Villigen, Switzerland. Tel.: + 41563105307.

E-mail address: shuang.wang@psi.ch (S. Wang).
}

cations on $\mathrm{Cu}^{2+}(S=1 / 2)$ sites have been found to induce disordered magnetism or even antiferromagnetic long range order (LRO) at Neél temperatures $T_{N}$ ranging from 3 to $8 \mathrm{~K}$ for $0.01<x<0.08$. Recent suggestions that dilution or bond-disorder could lead to new phenomena such as a Bose-glass phase [9], have renewed interest in spin ladders in general, and dilutable systems in particular.

Previous work on spin-ladder model materials, ranging from cuprates such as $\mathrm{Sr}_{14-x} \mathrm{Ca}_{x} \mathrm{Cu}_{24} \mathrm{O}_{41}$ and $\mathrm{SrCu}_{2} \mathrm{O}_{3}$, which represent the limits of strong magnetic exchange $J \sim 100 \mathrm{meV}$, to weakly coupled metal-organic compounds like $\left(\mathrm{C}_{5} \mathrm{H}_{12} \mathrm{~N}\right)_{2} \mathrm{CuBr}_{4}$ with $J \sim 1 \mathrm{meV}$ [10], left an important unexplored gap in the energy scales. A model material with intermediate energy scale $(J \sim 10 \mathrm{meV}$ ) would allow high quality mapping of the entire excitation spectrum, while still keeping the energy, field and temperature scales adequate for high resolution studies of finite temperature dynamics and effects of non-magnetic dilution. This inspired us to search for new experimental realizations of quantum spin ladder and investigate the impurity effects on them. The $\mathrm{BiCu}_{2} \mathrm{PO}_{6}$ compound was identified as a good candidate for such a spin ladder model material with intermediate energy scale. Magnetic susceptibility and heat capacity measurement on polycrystalline samples showing existence of a $\sim 34 \mathrm{~K}$ spin-gap were analyzed to imply leg-coupling $J_{1} / k_{B}=80 \mathrm{~K}$ and leg to rung 

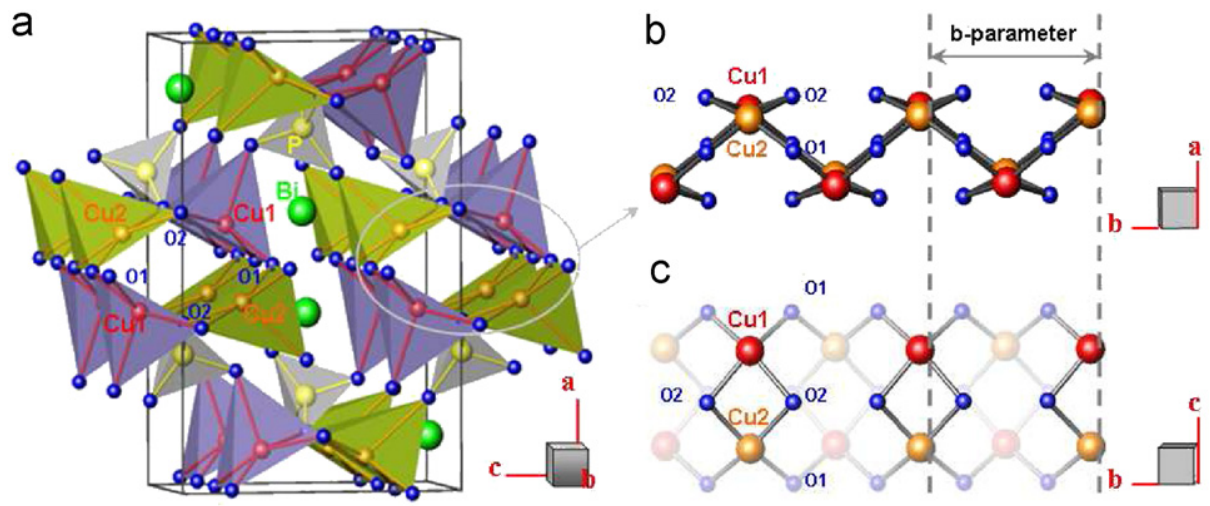

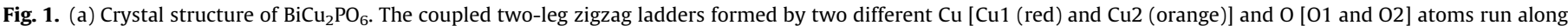

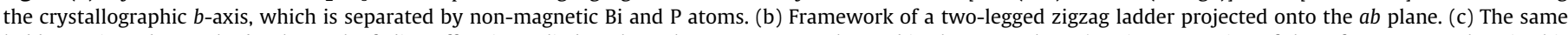

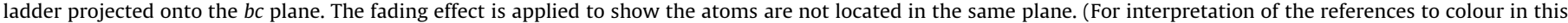
figure legend, the reader is referred to the web version of this article.)

exchange ratio $J_{1} / J_{2} \sim 1$ [11]. Furthermore, there might be frustrated coupling between adjacent rungs, which could add interesting new aspects to the spin ladder physics [12]. A schematic diagram of the structure is shown in Fig. 1a. Two edge-sharing $\mathrm{CuO}_{5}$ distorted square pyramids, with a $\mathrm{Cu}^{2+}$ ion at the center of the fivefold oxygen coordination, give rise to a $\mathrm{Cu}$ dimer with an intradimer distance of $2.8 \AA$. Each dimer connects two others by its four 01 corners resulting in a zigzag double chain (ladder) running along the $b$ axis (Fig. 1b). The interdimer cohesion is further strengthened by $\mathrm{PO}_{4}$ tetrahedra that connect two consecutive dimers by $\mathrm{O} 2$ corners. The $\mathrm{Bi}$ ions are positioned between two ladders (see Fig. 1a). The bond angle and bond length along the leg ( $\mathrm{Cu} 1-\mathrm{O} 1-\mathrm{Cu} 2)$ of the ladder are about $112^{\circ}$ and $3.2 \AA$ and along the rung ( $\mathrm{Cu} 1-\mathrm{O} 2-\mathrm{Cu} 2)$ the corresponding values are $92^{\circ}$ and $2.8 \AA$ [13]. The $\mathrm{BiCu}_{2} \mathrm{PO}_{6}$ structure has a promising chemical flexibility. Firstly, the vanadium analogue $\mathrm{BiCu}_{2} \mathrm{VO}_{6}$ and $\mathrm{P} / \mathrm{V}$-mixed $\mathrm{BiCu}_{2} \mathrm{P}_{1-x} \mathrm{~V}_{x} \mathrm{O}_{6}$ could be synthesized [14] and show an interesting incommensurate structural modulation along the ladders at vanadium doping level $x>0.7$. Secondly, Ca-doping at the Bi sites may introduces holes in the ladders [13]. Thirdly, the magnetic $\mathrm{Cu}^{2+}$ ions can be replaced by non-magnetic $\mathrm{Zn}^{2+}(S=0)$ [15] or magnetic $\mathrm{Ni}^{2+}(S=1)$ [16].

Most of the experiments reported to date were performed using polycrystalline samples. For further studies of the intrinsic nature of the ground state as well as the excited states in a large temperature range, i.e. for neutron inelastic scattering experiments, well below and above the gap temperature, large single crystals of high quality are indispensable, Mentre and Ketatni [14] obtained single crystals by a flux method, but the detailed procedure and the crystal size were not reported. In this work, the Travelling Solvent Floating Zone (TSFZ) method was applied for the crystal growth. Large centimeter size single crystals of $\mathrm{Bi}\left(\mathrm{Cu}_{1-x} \mathrm{Zn}_{x}\right)_{2} \mathrm{PO}_{6}(x=0 \%, 1 \%, 5 \%)$ were successfully grown and crystal quality, magnetic properties were investigated.

\section{Experimental procedure}

\subsection{Ceramics preparation}

Polycrystalline $\mathrm{Bi}\left(\mathrm{Cu}_{1-x} \mathrm{Zn}_{x}\right)_{2} \mathrm{PO}_{6}(x=0 \%, 1 \%, 5 \%)$ samples were prepared by a solid state reaction. Starting materials: $\mathrm{Bi}_{2} \mathrm{O}_{3}$ (CHEMPUR, 99.99\%), CuO (ALFA AESAR, 99.9999\%), and $\mathrm{NH}_{4} \mathrm{H}_{2} \mathrm{PO}_{4}$ (CHEMPUR, 99.999\%), ZnO (CHEMPUR, 99.999\%), were mixed in the desired stoichiometric proportions and annealed at 300, 500, $750{ }^{\circ} \mathrm{C}$ for $15 \mathrm{~h}$ at each step, and finally at $820^{\circ} \mathrm{C}$ for 2 days in alumina crucibles in air, with intermediate grindings. The total synthesis reaction of undoped $\mathrm{BiCu}_{2} \mathrm{PO}_{6}$ compound can be written as follows:

$$
\mathrm{Bi}_{2} \mathrm{O}_{3}+4 \mathrm{CuO}+2 \mathrm{NH}_{4} \mathrm{H}_{2} \mathrm{PO}_{4} \rightarrow 2 \mathrm{BiCu}_{2} \mathrm{PO}_{6}+2 \mathrm{NH}_{3}+3 \mathrm{H}_{2} \mathrm{O}
$$

The resulting bright green sintered material of $\mathrm{BiCu}_{2} \mathrm{PO}_{6}$ was powderised again and then loaded into a rubber tube and pressed hydrostatically at $4 \mathrm{Kbar}$ to produce rods of $5-8 \mathrm{~mm}$ in diameter and $95 \mathrm{~mm}$ in length. These rods were subsequently sintered at $840{ }^{\circ} \mathrm{C}$ in air for $24 \mathrm{~h}$. The rods were then pre-annealed in $\mathrm{O}_{2}$ at temperature close to the melting point by using the image furnace used for the crystal growth, in order to obtain the highest possible density. Sintered rods with about $95 \%$ of the crystallographic density $\left(d_{x}=6.481 \mathrm{~g} / \mathrm{cm}^{3}[17]\right)$ were obtained, which in this case was found to be sufficient. The rod color changed from green to partly shining gray, with matte surface after the final annealing. According to our preliminary differential thermal analysis experiment (NETSCH STA 449C), the $\mathrm{BiCu}_{2} \mathrm{PO}_{6}$ melts incongruently at $896( \pm 5){ }^{\circ} \mathrm{C}$. The $\mathrm{BiCu}_{2} \mathrm{PO}_{6}, \mathrm{Cu}_{2} \mathrm{O}$ and $\mathrm{BiPO}_{4}$ phases were found for the melted undoped sample by the XRD phase analysis.

\subsection{Crystal growth of $\mathrm{Bi}\left(\mathrm{Cu}_{1-x} \mathrm{Zn}_{x}\right)_{2} \mathrm{PO}_{6}(x=0 \%, 1 \%, 5 \%)$}

Single crystals of $\mathrm{BiCu}_{2} \mathrm{PO}_{6}$ were grown by the TSFZ technique in an image furnace (Model FZ-T-10000-H-VI-VP, Crystal System. Inc., Japan) using four $300 \mathrm{~W}$ halogen lamps as heat sources. A number of preliminary tests have been performed to find the proper growth conditions. Several trials with different growth rates $(0.5-2 \mathrm{~mm} / \mathrm{h})$ were carried out. A growth process instability accompanied by a volume change of the molten zone was observed at growth rates above $1.5 \mathrm{~mm} / \mathrm{h}$ (see Fig. 2). Additionally, crystals grown at rates above $2 \mathrm{~mm} / \mathrm{h}$ always consist of at least three domains, which preserve along the whole length of the crystal rod (see Fig. 6a). Single domain growth could be achieved only by growing at a relatively low rate (slower than $1 \mathrm{~mm} / \mathrm{h}$ ). Additionally, it was found that decreasing the diameter of the feed rod from $8 \mathrm{~mm}$, down to $5 \mathrm{~mm}$, considerably improves the molten zone stability and allows growth of crackfree crystals up to $50 \mathrm{~mm}$ in length. Significant evaporation of the material were not observed during crystal growth. The quartz tube were only slightly covered after all performed growth experiments.

The optimal conditions for the $\mathrm{BiCu}_{2} \mathrm{PO}_{6}$ crystal growth are an atmosphere $20 \% \mathrm{O}_{2}$ in $\mathrm{Ar}$ gas at a pressure of 6 bar, growth rate of $1 \mathrm{~mm} / \mathrm{h}$ and a counter-rotation of the upper and lower rods of 


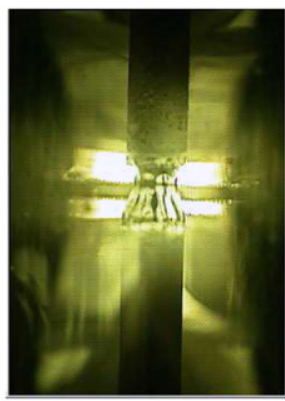

Start

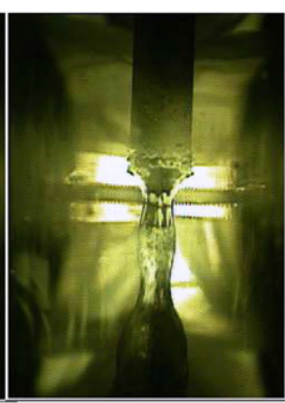

After 12h

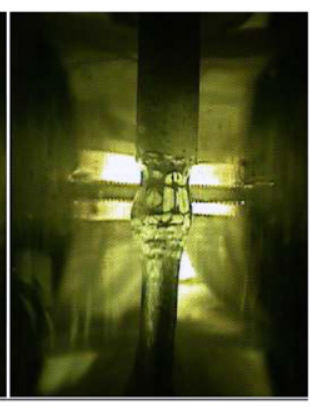

After 40h
Fig. 2. A growth process instability caused by the change of the liquid volume at the molten zone was observed at growth rates above $2 \mathrm{~mm} / \mathrm{h}$.

$20 \mathrm{rpm}$, to secure the liquid homogeneity as well as the homogeneous temperature distribution within the liquid zone. Since polycrystalline seed and feed rods were used in this work, the as-grown material only became single crystalline after $1.5-2.5 \mathrm{~cm}$ of the growth. At the beginning i.e. after several $\mathrm{mm}$ of growth, the crystals exhibited bark-like edges and smooth, shiny facets around the rod. In the course of the growth, two facets increase in size and expel the other until the crystal becomes single domain over the whole cross-section.

The $\mathrm{Zn}$-doped $\mathrm{BiCu}_{2} \mathrm{PO}_{6}$ crystals were grown following the same routine as for the undoped ones. To our knowledge no $\mathrm{Zn}$-doped single crystals have been grown up to now although some work on ceramics has been done by Koteswararao et al. [13]. In many cases, the introduction of additional cations into the system drastically changes the crystal growth conditions. This was observed previously e.g. in $\mathrm{SrCu}_{2}\left({ }^{11} \mathrm{BO}_{3}\right)_{2}$ system [18]. Surprisingly, in the case of the $\mathrm{BiCu}_{2} \mathrm{PO}_{6}$ system, we successfully substituted $\mathrm{Zn}^{2+}$ (ionic radii $0.68 \AA$ ) for $\mathrm{Cu}^{2+}$ (ionic radii $0.65 \AA$ [19]) at different Zn-doping level $(x=1 \%, 5 \%)$ without any necessary adjustment of the growth conditions. The growth processes were very smooth and several $70 \mathrm{~mm}$ long single crystal of $\mathrm{Bi}\left(\mathrm{Cu}_{1-x} \mathrm{Zn}_{x}\right)_{2} \mathrm{PO}_{6}(x=1 \%, 5 \%)$ were obtained. However, our attempts to grow large $\mathrm{BiZn}_{2} \mathrm{PO}_{6}(x=100 \%)$ crystals failed and material with multiple grains and irregular shape was obtained. The failure is probably due to the fact that $\mathrm{BiZn}_{2} \mathrm{PO}_{6}$ undergoes a structural phase transition at $325^{\circ} \mathrm{C}$, which will create cracks or/ and twinning. Multiple grains and irregular shape at growth temperature is (probably) caused by presence of two phases due to incongruent melting [19].

\section{Characterization and magnetic properties}

Photographs of the as-grown $\mathrm{Bi}\left(\mathrm{Cu}_{1-x} \mathrm{Zn}_{x}\right)_{2} \mathrm{PO}_{6}(x=0 \%, 1 \%, 5 \%)$ crystals, are presented in Fig. 3 , together with the white rod of $\mathrm{BiZn}_{2} \mathrm{PO}_{6}(x=100 \%)$. Crystals with different $\mathrm{Zn}$-doping level were cylinder shaped, opaque, about 40-60 mm long, with glossy surface and shining stripes and facets. The density of the $\mathrm{BiCu}_{2} \mathrm{PO}_{6}$ crystal $\left(d_{\text {crystal }}=6.48 \pm 0.02 \mathrm{~g} / \mathrm{cm}^{3}\right)$, determined by buoyancy method, is in a very good agreement with the crystallographic density $\left(6.481 \mathrm{~g} / \mathrm{cm}^{3}\right)$. The phase analysis and lattice parameter study were performed at room temperature using a X-ray powder diffractometer Bruker D8, in $\Theta: 2 \Theta$ mode. Scans from $10^{\circ}$ to $100^{\circ}$ with a step of $0.0092^{\circ}$. For each crystal, a piece from crystal rod top and bottom was crushed and Rietveldt structure refinement analyses were performed, individually. All samples were found to be single phase with no traces of additional phases. The refinement pattern of the pure $\mathrm{BiCu}_{2} \mathrm{PO}_{6}$ sample is shown in Fig. 4. The crystal symmetry at room temperature was found to be orthorhombic with Pnma space group and lattice constants: $a=11.7768(1) \AA$,

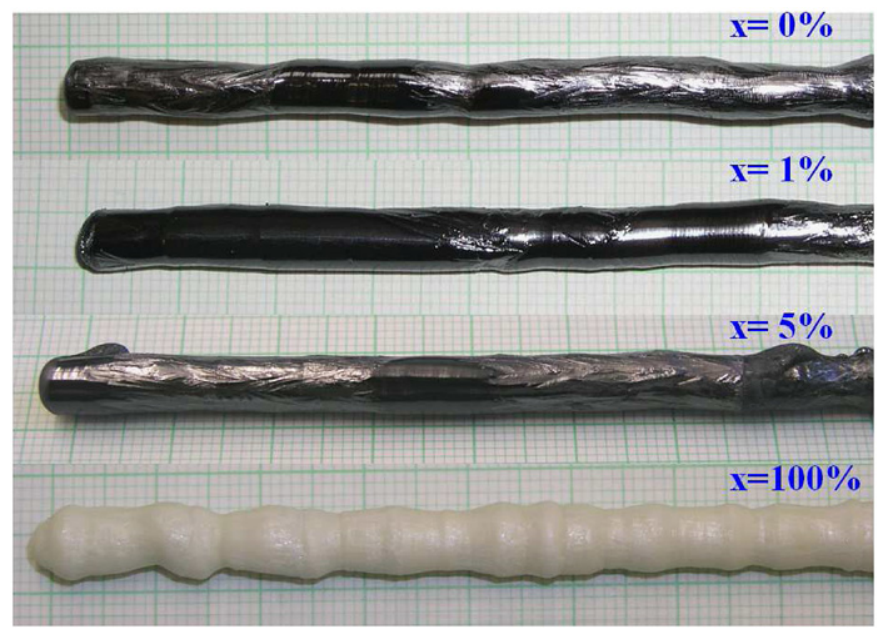

Fig. 3. As-grown single crystals of $\mathrm{Bi}\left(\mathrm{Cu}_{1-x} \mathrm{Zn}_{x}\right)_{2} \mathrm{PO}_{6}(x=0 \%, 1 \%, 5 \%)$, together with rod of $\mathrm{BiZn}_{2} \mathrm{PO}_{6}$.

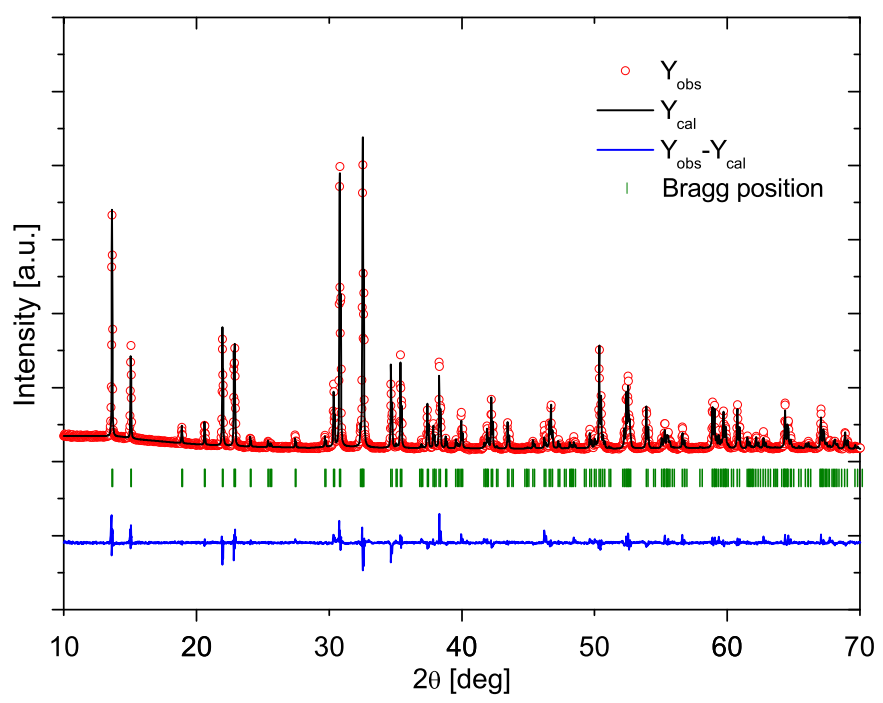

Fig. 4. The $\mathrm{X}$-ray diffraction pattern from crushed single crystal of $\mathrm{BiCu}_{2} \mathrm{PO}_{6}$ is shown together with the Rietveldt refinement using FULLPROF. The red open circles indicate the experimental XRD data, the solid black line is calculated based on the refinement, green vertical short lines are the Bragg positions and the blue line is the difference of measured and calculated data. (For interpretation of the references to colour in this figure legend, the reader is referred to the web version of this article.)

$b=5.1729(0) \AA, c=7.7900(1) \AA$, which are in reasonable agreement with previously reported data [11]. The XRD patterns for Zn-doped samples were also indexed based on Pnma space group. The XRD peaks shift towards smaller $2 \Theta$ angles as the $\mathrm{Zn}$ content increase from $x=0 \%$ to $5 \%$, as shown in Fig. 5 . This is indicative of $\mathrm{Zn}^{2+}$ cations incorporation into the $\mathrm{Cu}^{2+}$ sites. The lattice parameters $a, b$ and $c$ from the refinement of all samples with different $\mathrm{Zn}$-doping content $(x)$ are shown in Table 1.

Morphology of the $\mathrm{BiCu}_{2} \mathrm{PO}_{6}$ crystal sample was checked by optical microscope observation of the polished cross-section parallel and vertical to the growth direction. As shown in the Fig. 6a, single crystal samples grown with the rate of $1 \mathrm{~mm} / \mathrm{h}$ consist of only one domain. For comparison, a sample grown with the rate of $2 \mathrm{~mm} / \mathrm{h}$ shows three domains, pores and cracks (Fig. 6a right). The X-ray Laue and neutron diffraction techniques were used to assess the crystal quality as well as to determine the orientation of the grown crystals. As shown in Fig. 6b, two identical ( $\left.\begin{array}{lll}2 & 0 & 0\end{array}\right)$ diffraction patterns were observed at both ends of 


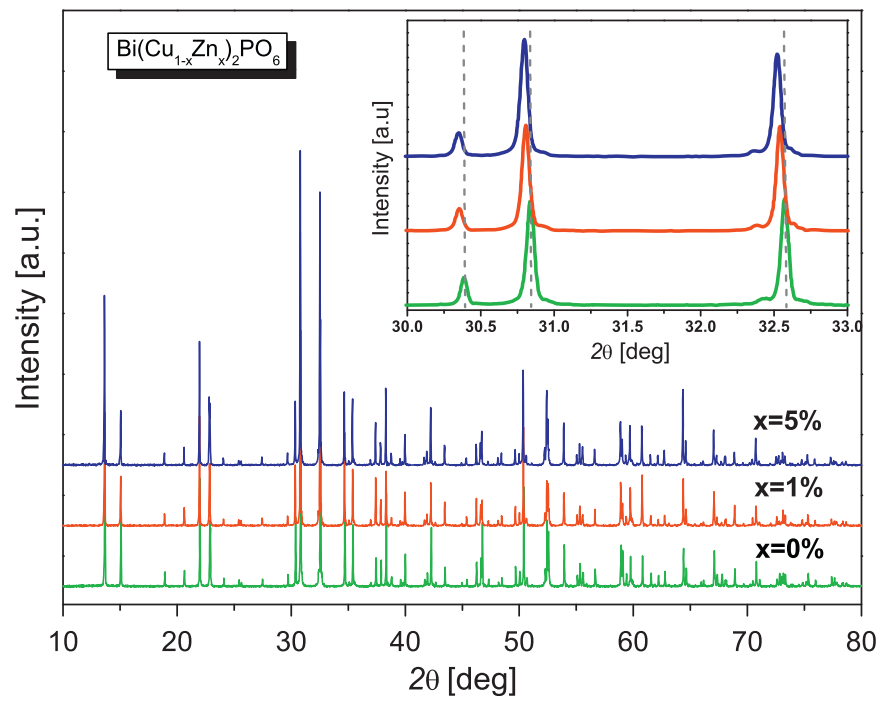

Fig. 5. XRD pattern of the crushed single crystals of $\mathrm{Bi}\left(\mathrm{Cu}_{1-x} \mathrm{Zn}_{x}\right)_{2} \mathrm{PO}_{6}(x=0 \%, 1 \%$, $5 \%)$. Inset: a part of XRD pattern is enlarged for clarity. The peaks shift towards smaller $2 \Theta$ for $\mathrm{Zn}$ doping up to $5 \%$, which is a clear indication of a change in lattice parameters and $\mathrm{Zn}$ incorporation into the structure.

Table 1

Lattice parameters and unit cell volume (s.g. Pnma) for four $\mathrm{Bi}\left(\mathrm{Cu}_{1-x} \mathrm{Zn}_{x}\right)_{2} \mathrm{PO}_{6}$ samples with $\mathrm{Zn}$ concentrations $x=0,1,5$ and $100 \%$.

\begin{tabular}{lllll}
\hline Compound & $a(\AA)$ & $b(\AA)$ & $c(\AA)$ & Volume $\left(\AA^{3}\right)$ \\
\hline $\mathrm{BiCu}_{2} \mathrm{PO}_{6}$ & $11.7768(1)$ & $5.1729(0)$ & $7.7900(1)$ & 474.56 \\
$\mathrm{BiCu}_{0.99} \mathrm{Zn}_{0.01} \mathrm{PO}_{6}$ & $11.7800(1)$ & $5.1742(1)$ & $7.7924(1)$ & 474.96 \\
$\mathrm{BiCu}_{0.95} \mathrm{Zn}_{0.05} \mathrm{PO}_{6}$ & $11.7822(1)$ & $5.1770(1)$ & $7.7969(1)$ & 475.61 \\
$\mathrm{BiZn}_{2} \mathrm{PO}_{6}[19]$ & $11.8941(3)$ & $5.2754(2)$ & $7.8161(2)$ & 490.44 \\
\hline
\end{tabular}

the sample, which confirm that one single domain extends along the entire crystal. Contrary to X-ray Laue, which only probes the surface, neutrons penetrate the crystal and therefore probe the bulk properties. The bulk quality of the $\mathrm{BiCu}_{2} \mathrm{PO}_{6}$ crystal was confirmed by neutron rocking curves (performed on Morpheus, PSI, Switzerland) shown in Fig. 6c. The strong (2 00 ) Braggreflection display a symmetrical width of $0.51^{\circ}$ (FWHM), which is limited only by the instrumental resolution (around $0.2^{\circ}$ ), indicating good bulk crystal quality.

The magnetic properties of all crystal samples with different doping levels were measured as a function of temperature from $300 \mathrm{~K}$ down to $2 \mathrm{~K}$ using a SQUID magnetometer (Quantum Design). The temperature dependent susceptibility $\chi(T)=M(T) / H$ was determined with a magnetic field of 1000 Oe applied in the $b c$ plane (See Fig. 7). It is evident that all crystals share the same general features. Upon decreasing temperature, $\chi(T)$ increases towards a broad maximum around $60 \mathrm{~K}$, characteristic of lowdimensional antiferromagnets. The subsequent rapid exponential decrease bear witness of a gapped ground state as expected for a spin ladder.

At the lowest temperatures a Curie-like upturn, reflecting weakly coupled spins, increases with $\mathrm{Zn}$ content. This component can contain contributions both from $\mathrm{Cu}^{2+}$ spins adjacent to a vacancy due to a non-magnetic $\mathrm{Zn}^{2+}$ ion in the ladders, and from impurity moments. The $\mathrm{Cu}$-free $x=100 \% \mathrm{Zn}$ sample also show a low-temperature upturn, which is well described by a $0.1 \%$ fraction of completely uncorrelated paramagnetic moments. Assuming a similar contribution in the $\mathrm{Cu}$-based samples accounts for most of the upturn observed in the pure $\mathrm{Cu}$ sample,
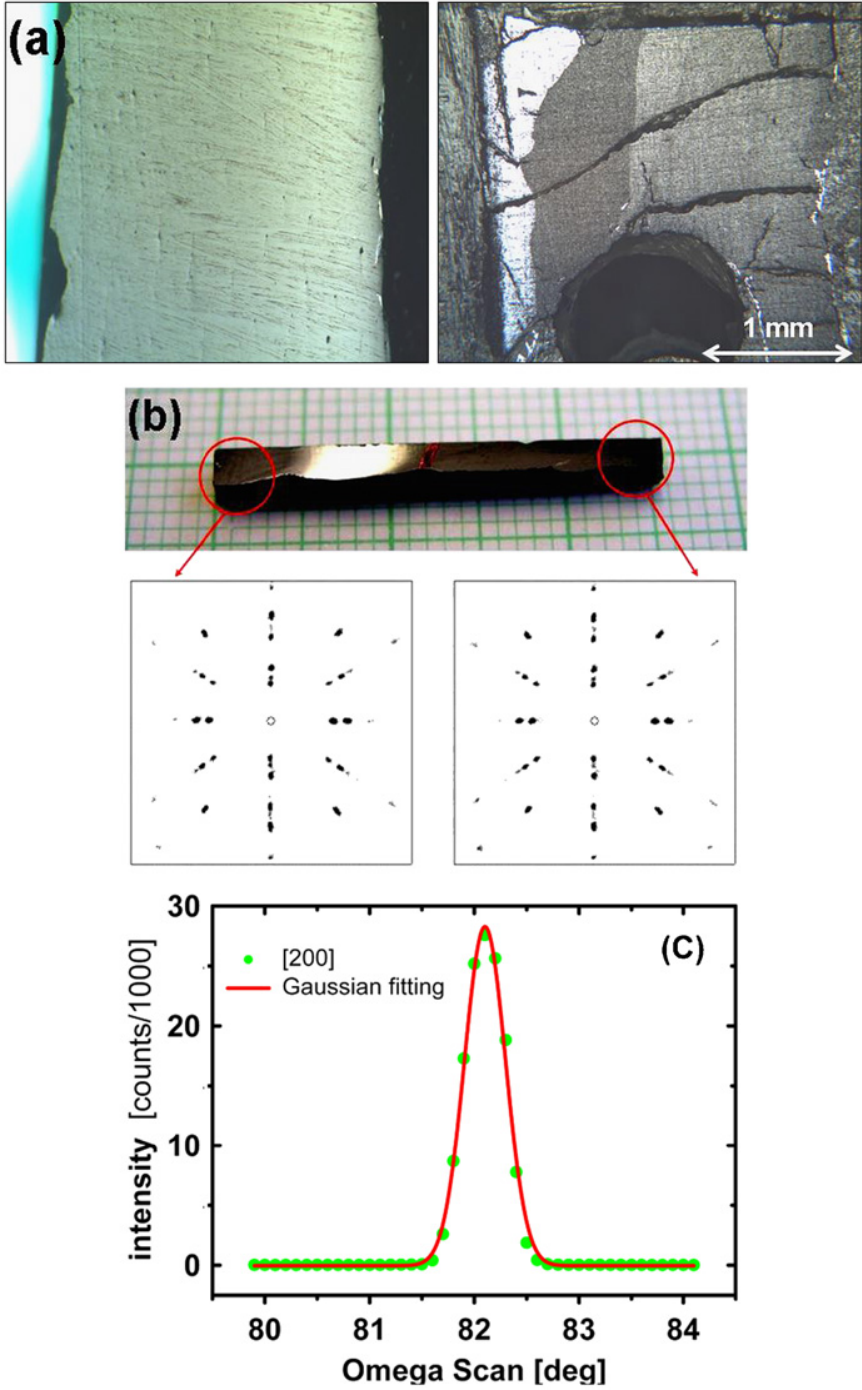

Fig. 6. (a) Optical microscope photographs (under polarized light) of polished cross-section parallel to the growth axis of a single domain crystal rod, (left), and for comparison, a sample grown at the rate of $2 \mathrm{~mm} / \mathrm{h}$, (right). (b) $\mathrm{BiCu}_{2} \mathrm{PO}_{6}$ single crystal sample and X-ray back-scattering Laue pictures taken along $a$-axis $\left( \pm 1^{\circ}\right)$ recorded by single translation from both ends of the undoped sample (c) Neutron rocking curve of the (200) Bragg reflection of undoped single crystal sample carried out at Morpheus, PSI $(\lambda=4.3 \AA)$.

and allow us to quantify the amount of weakly coupled $\mathrm{Cu}^{2+}$ spins as function of doping. Subtracting the curve measured on the pure $\mathrm{Zn}$ sample from those measured on the Cu samples does not result in purely paramagnetic tails [11]. Therefore the latter were fitted to the sum of a Curie-Weiss term $C /(T-\Theta)$ and an exponentially activated ladder contribution. The obtained concentrations of weakly coupled spins, $0.15 \%, 1.4 \%$, and $5.6 \%$, rather closely match the nominal Zn-doping levels. Furthermore, the very low defect concentration in the nominally undoped crystal implies coherence lengths exceeding several hundred sites along the ladder, allowing for intricate studies of the quantum dynamics in these samples.

\section{Conclusions}

In summary we have presented the details of the crystal growth, crystal quality and magnetic properties of the incongruently melting $\mathrm{Bi}\left(\mathrm{Cu}_{1-x} \mathrm{Zn}_{x}\right)_{2} \mathrm{PO}_{6}(x=0 \%, 1 \%, 5 \%)$. Large single 


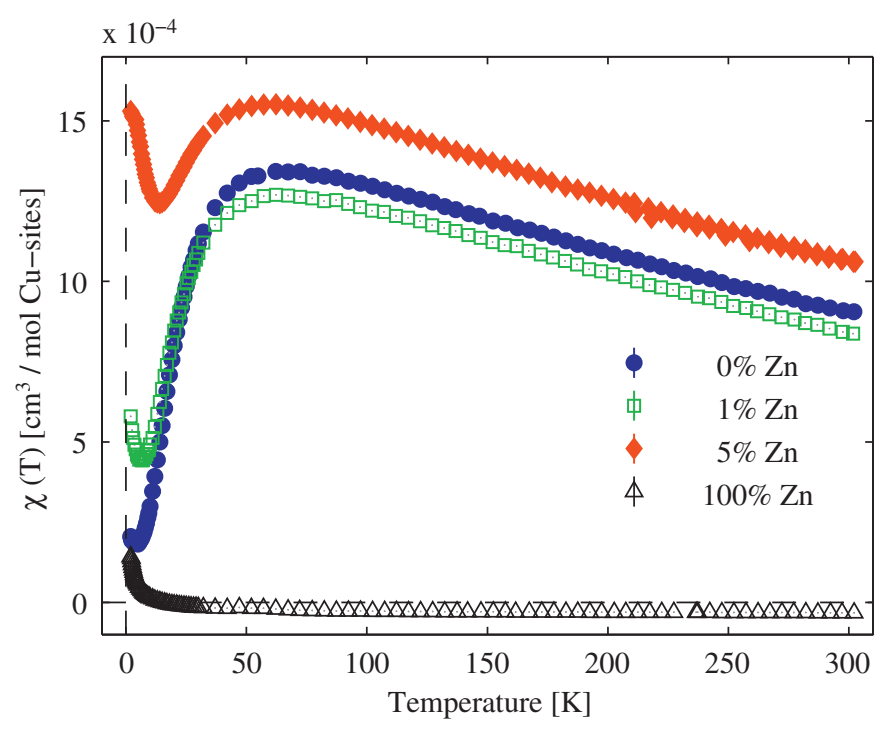

Fig. 7. Magnetic susceptibility $\chi(T)$ for four $\mathrm{Bi}\left(\mathrm{Cu}_{1-x} \mathrm{Zn}_{x}\right)_{2} \mathrm{PO}_{6}$ samples with $\mathrm{Zn}$ concentrations $x=0,1,5$ and $100 \%$.

crystals were achieved using Travelling Solvent Floating Zone technique at a relatively slow speed of $1 \mathrm{~mm} / \mathrm{h}\left(20 \% \mathrm{O}_{2}\right.$ in $\mathrm{Ar}$ gas $)$ at a pressure of 6 bar. Our attempt of 100\% Zn-doping crystal growth failed, probably due to structural phase transition at $325^{\circ} \mathrm{C}$. High phase purity and crystalline quality were demonstrated by XRD structure analysis, X-ray Laue and Neutron diffraction techniques. Magnetic susceptibility measurements confirmed a very low impurity level of the undoped crystal and the expected generation of un-paired spins by the $\mathrm{Zn}$-doping. Crystals of such quality as presented in this work can be applied for further investigations of the dispersion relation of the triplet excitations and the dynamics of the excess spins induced by $\mathrm{Zn}$-doping and substitution effect on the ladder gap in this compound family.

\section{Acknowledgements}

We acknowledge F. Casola, M. Mourigal, M. Bendele, R. Frison, M. Mederde, C. Marcelot and M. Kenzelmann for their kind help and discussion on the related measurement at ETHZ, PSI and ILL. This work is supported by the Swiss National Science Foundation on the project of dimer physics and NCCR MaNEP.

\section{References}

[1] A.W. Sandvik, Phys. Rev. B 56 (1997) 11678.

[2] P. Abbamonte, G. Blumberg, A. Rusydi, A. Gozar, P.G. Evans, T. Siegrist, L. Venema, H. Eisaki, E.D. Isaacs, G.A. Sawatzky, Nature 431 (2004) 1078.

[3] H.M. Rønnow, D.F. McMorrow, R. Coldea, A. Harrison, I.D. Youngson, T.G. Perring, G. Aeppli, O. Syljuåsen, K. Lefmann, C. Rischel, Phys. Rev. Lett. 87 (2001) 037202.

[4] K.P. Schmidt, G.S. Uhrig, Phys. Rev. Lett. 98 (2003) 087202.

[5] T. Giamarchi, Ch. Rüegg, O. Tchernyshyov, Nature Phys. 4 (2008) 198.

[6] E. Dagotto, T.M. Rice, Science 271 (1996) 618.

[7] M. Uehara, T. Nagata, J. Akimitsu, H. Takahasi, N. Mori, K. Kinoshita, J. Phys. Soc. Jpn. 65 (1996) 2764.

[8] M. Azuma, Y. Fujishiro, M. Takano, M. Nohara, H. Takagi, Phys. Rev. B 55 (1997) 8658.

[9] O. Nohadini, S. Wessel, S. Haas, Phys. Rev. Lett. 95 (2005) 227201.

[10] B. Thielemann, Ch. Rüegg, H.M. Rønnow, A.M. Läuchli, J.S. Caux, B. Normand, D. Biner, K.W. Krämer, H.U. Güdel, J. Stahn, K. Habicht, K. Kiefer, M. Boehm, D.F. McMorrow, J. Mesot, Phys. Rev. Lett. 102 (2009) 107204.

[11] B. Koteswararao, S. Salunke, A.V. Mahajan, I. Dasgupta, J. Bobroff, Phys. Rev. B 76 (2007) 052402.

[12] O. Mentré, E. Janod, P. Rabu, M. Hennion, F. Leclercq-Hugeux, J. Kang, C. Lee, M.-H. Whangbo, S. Petit, Phys. Rev. B 80 (2009) 180413R.

[13] B. Koteswararao, A.V. Mahajan, L.K. Alexander, J. Bobroff, J. Phys. Condens. Matter 22 (2010) 035601.

[14] O. Mentré, E.M. Ketatni, M. Colmont, M. Huvé, F. Abraham, V. Petricek, J. Am. Chem. Soc. 128 (2006) 10857.

[15] J. Bobroff, N. Laflorencie, L.K. Alexander, A.V. Mahajan, B. Koteswararao, P. Mendels, Phys. Rev. Lett. 103 (2009) 047201.

[16] L.K. Alexander, J. Bobroff, A.V. Mahajan, B. Koteswararao, N. Laflorencie, F. Alet, Phys. Rev. B 81 (2010) 054438.

[17] F. Abraham, M. Ketatni, G. Mairesse, B. Mernari, Eur. J. Solid. Inorg. Chem. 31 (1994) 313.

[18] H.A. Dabkowska, A.B. Dabkowski, G.M. Luke, S.R. Dunsiger, S. Haravifard, M. Cecchinel, B.D. Gaulin, J. Crystal Growth 306 (2007) 123.

[19] E.M. Ketatni, B. Mernari, F. Abraham, O. Mentre, J. Solid State Chem. 153 (2000) 48. 